\title{
EDITORIAL
}

\section{Prevention of Sudden Cardiac Death (SCD) - Possible Strategies}

Sudden cardiac death (SCD) causes millions of deaths each year in world. Sudden implies that death occurred unexpectedly and that the sequence of events that led to death occurred within a short time span, usually one hour before death. It is generally assumed that the majority (85\%) of SCD victims die of ventricular tachyarrhythmias, usually in the form of ventricular fibrillation (VF). The remaining SCD victims die either of bradyarrhythmias or of acute pump failure.

SCD may be the first clinical manifestation of coronary artery disease in as many as one in five coronary heart disease patients.SCD urgently requires primary prevention because the first clinical event is often fatal, especially in patients with ventricular tachyarrhythmias. Patients with acute bradyarrhythmias often retain a basal circulation due to ventricular escape rhythms and appropriate medical treatment or a pacemaker can be deployed in time to prevent irreversible organ damage. On the other hand VF results in a rapid and complete loss of blood circulation and If left untreated, this condition results in irreversible organ (most notably brain) damage after a few minutes.Even in regions of the world with highly developed emergency medical care systems, only a small proportion of patients suffering from VF will leave the hospital alive.Primary prevention of SCD is therefore in part a diagnostic challenge- that is, it requires identification of future sudden death victims before the first arrhythmia episode.

Secondary prevention refers to clinical intervention in patients who have survived a prior cardiac arrest or sustained ventricular tachycardia. In these patients, the ICD has proven superior to antiarrhythmic drug therapy for prolonging survivalSecondary prevention refers to clinical intervention in patients who have survived a prior cardiac arrest or sustained ventricular tachycardia.In these patients, the ICD has proven superior to antiarrhythmic drug therapy for prolonging survivalCorrect identification of future SCD victims is especially important as there is an effective treatment, namely defibrillation via an external or internal (implanted) defibrillator. More recently, their effectiveness has been demonstrated for primary prevention of SCD in patients with severely reduced left ventricular function, caused either by coronary artery disease or by cardiomyopathies.

In addition, several drugs(aspirin, "statins", â blockers, ACEI, Amiodarone) are useful to prevent SCD in patients with known coronary artery disease or heart failure. Besides,cardiac resynchronisation therapy (CRT) by biventricular stimulation may decrease sudden death rates in heart failure patients.

A simple and powerful clinical tool to identify patients with inherited arrhythmogenic diseases is an appropriate family history of sudden death. Sudden deaths before the age of 60 in first degree relatives, but also unexplained "accidents" for example, car crashes without the involvement of alcohol, bad weather conditions or other explanations for the crash should trigger further investigations. In addition, the standard ECG can identify some patients with inherited arrhythmogenic diseases Other diseases-for example, familial hypertrophic cardiomyopathy or arrhythmogenic right ventricular cardiomyopathy-may require echocardiography and further cardiac investigations to be diagnosed correctly.

Thus prevention of SCD can be achieved through four types of interventions:

1) prevention of acute ischaemic events by prevention of coronary artery disease

2) optimal treatment of heart failure and protection of high risk patients with a defibrillator

3) identification of an inherited risk for arrhythmias in the surface ECG and subsequent treatment of the underlying disease

4) prudent use of drugs with a potential for proarrhythmic side effects.

Future diagnostic tests and therapeutic interventions will be based on a more comprehensive understanding of the cellular and electrical events that trigger SCD. Future disease or gene specific interventions may, in the future, help to prevent SCD in patients with inherited arrhythmogenic disorders.
Dr. Mohammad Safiuddin
Associate Professor of Cardiology
Bangabandhu Sheikh Mujib Medical University, Dhaka
E-mail: safiuddin1960@yahoo.com 